\title{
Educación Física en Venezuela: Pertinencia teórica y viabilidad de la reforma del currículo 2016-2018
}

\author{
Jesús Navarro \\ Universidad Pedagógica Experimental Libertador, Venezuela \\ jesusn35@gmail.com \\ Carlyla Ramos \\ Universidad Pedagógica Experimental Libertador, \\ Venezuela \\ Carmen Varguillas \\ Universidad Pedagógica Experimental Libertador, \\ Venezuela
}

Physical Education in Venezuela: Theoretical and feasibility relevance of the curriculum reform 2016-2018

\begin{abstract}
Resumen:
La finalidad de este artículo fue Analizar la pertinencia teórica y viabilidad de la reforma del currículo del nivel de media general en el área de Educación Física en Venezuela 2016-2018. El presente estudio, inédito y sistemático permitió identificar las consecuencias de esta posible aprobación. Para ello se realizó una investigación documental sobre estudios referenciales en el ámbito internacional y nacional, documentos y opiniones de especialistas. A manera de conclusión: el área de Educación Física adquiere beneficios en relación al aumento de horas y sesiones de clases para cada sección, disponiendo más tiempo para el desarrollo integral del educando, sin embargo existen otros elementos importantes que obstaculizan este proceso educativo, desde el especialista del área hasta las instalaciones deportivas. Se recomienda al ente rector que estudien estas expectativas de lo contrario esta reforma sería un fracaso y debilitaría aún más el sistema educativo venezolano.
\end{abstract}

Palabras Clave: Nivel media general, Educación física, Reforma del currículo, Pertinencia teórica.

\begin{abstract}
:
The purpose of this article is to analyze the theoretical and feasibility relevance of the curriculum reform of the general average level in the area of Physical Education in Venezuela 2016-2018. The present, unpublished and systematic study allowed identifying the consequences of this possible approval. For this purpose, a documentary research on referential studies in the international and national scope, documents and opinions of specialists was carried out. As a conclusion, we can say that the area of Physical Education acquires benefits in relation to the increase of hours and class sessions for each section, providing more time for the integral development of the student. However, there are other important elements that hinder this educational process, from the specialist of the area up to the sports facilities. It is recommended to the governing body to study these expectations otherwise this reform would be a failure and would further weaken the Venezuelan educational system.
\end{abstract}

KEYWORDS: General average level, Physical education, Reform of the curriculum, Theoretical.

\section{INTRODUCCIÓN}

En este mundo globalizado subyacen prioridades que cada país debe asumir para estar en concordancia con los avances y dinámicas que son necesarias para sus ciudadanos. En primer lugar, se puede señalar el ámbito económico como elemento fundamental de este fenómeno mundial, ya que este aspecto permite el intercambio de bienes y servicios entre los pueblos, independientemente de su idiosincrasia. El otro aspecto 
importante es el sistema educativo que implementan los países para formar a sus ciudadanos con las nuevas tendencias educativas e intereses del Estado que correspondan con los principios que se declaran en su Constitución Nacional, además que se acerquen a los lineamientos de las estructuras continentales como la Organización de las Naciones Unidas (ONU), Organización de las Naciones Unidas para la Educación, la Ciencia y la Cultura (UNESCO), Mercado Común del Sur (MERCOSUR), Organización Mundial para la Salud (OMS) para ir en reciprocidad con sus vecinos. Esto permite conformar un entorno equilibrado y sustentable en beneficio de todos los individuos que hacen vida en este planeta. Para corroborar esta apreciación, Lamogglia de Ramírez (2012), señala:

Los sistemas educativos son influenciados por los cambios sociales, de allí que los mismos deban ajustarse, reajustarse o redefinirse con cierta frecuencia, con vista a los adelantos y cambios de paradigmas que ocurren en el mundo social, pues hay necesidad de orientar los niveles del sistema educativo hacia la integración del hombre en su desarrollo personal, social y laboral (p. 15).

Por lo anteriormente mencionado, es necesario mirar hacia otras fronteras para distinguir los aciertos y desaciertos en cuanto a la aplicabilidad de las políticas educativas implementadas, consensuando las que son prósperas y que arrojen el mayor índice de progreso. Sin embargo, el elemento económico y educativo no son los únicos aspectos a considerar para satisfacer las necesidades de una comunidad, se hace imperiosa la atención en otras áreas que demandan los ciudadanos, tales como lo declaró desde hace un tiempo la Organización de las Naciones Unidas (1948): "salud, alimentación y nutrición, educación, condiciones de trabajo, condiciones de empleo, consumo y ahorro de la colectividad, transporte, habitación, vestido, recreación y entretenimiento, seguridad social y libertad humana" (p. 340), tópicos que esta organización considera prioritaria, aunque dicta de muchos años atrás, aún siguen siendo las debilidades de las naciones en estos momentos.

Estas son en su mayoría, las condiciones mínimas que debe disponer un gobierno para que sus ciudadanos puedan vivir en una situación de bienestar, produciendo un estado de ánimo óptimo, dedicándose el individuo plenamente al desarrollo y porvenir de su país.

Sin embargo, desde el año 2016, todos los países están atravesando por una situación difícil y compleja, descuidando las áreas que se describieron anteriormente. Para corroborar la apreciación de los autores de esta investigación, se puede mencionar lo señalado por Ban Ki-Moon en (ONU, 2015),

...actualmente el mundo vive la crisis más aguda en las relaciones internacionales de la historia de la ONU...la crisis se enmarca en el contexto de varios conflictos regionales, el extremismo, el terrorismo, la pobreza y varias epidemias...los líderes de Estado deben aprender a gobernar con participación de todas las capas sociales (p. s/n).

Este panorama mundial no excluye a ningún país, la seguridad social de cada región está impregnada de esta crisis, los más ricos oprimen a los más pobres, el populismo como estrategia de obtener el poder está ganando la batalla a los líderes con una visión de país basada en una política de Estado con tendencia republicana, donde el ciudadano sea el centro de atención.

Sin embargo, aún no se ha llegado a resolver dicha situación, como ejemplo de ello, se debe describir la perspectiva de Venezuela como contexto propio de este artículo, nación suramericana que no escapa de esta problemática. En este sentido, se presentará de forma genérica los elementos que de alguna manera relatan el escenario que se vive actualmente en este país. Al respecto, Linarez (2016), puntualiza:

El 2016 abre el escenario político en Venezuela con una crisis económica profunda con responsabilidades compartidas...convulsionado e inmerso en un sinfín de problemas, circunstancias y procesos geopolíticos por el control del poder...El desabastecimiento, la inflación, la inseguridad, el alto costo de la vida son los principales problemas que viven y padecen diariamente los venezolanos y las venezolanas sin que el gobierno parezca encontrar una solución real y definitiva a estos problemas...La población pasa gran parte de su tiempo en largas y tormentosas colas para tratar de adquirir los productos de primera necesidad, no pueden acceder a gran parte de los productos en general porque la inflación, la especulación se consumen sus sueldos, sus logros sociales y su baja calidad de vida...se entiende porque la oposición ha vuelto al poder y porque ha aumentado la migración de venezolanos y venezolanas hacia el exterior en estos últimos 3 años (p.s/n). 
Adicionalmente, se puede acotar otros elementos, la corrupción, el narcotráfico y la delincuencia están a la orden del día, la vivienda y los servicios están inalcanzables, la hiperinflación se acerca al $600 \%$ para finales del 2017 según el diputado de la Asamblea Nacional y economista José Guerra. En este orden Rodríguez (citado en Linarez, 2016), jefe de la empresa Torino Capital, prevé que "la inflación en la economía aumente en el 2018 a $10.000 \%$ o $15.000 \%$, aspecto que vulnera cualquier intento de bienestar" (p. s/n). También es importante señalar que en los países latinoamericanos la inflación anual está entre el $2 \%$ a 4\%, cifra ideal para una vida sustentable y próspera (Finanza Digital, ob. cit.).

Aunado a esto, el ejecutivo venezolano decretó en el mes de diciembre del año 2016 eliminar el billete de bs. 100, el de más alta denominación para evitar la "supuesta" fuga de esa moneda al vecino país -Colombia-como una medida para combatir la sospechada guerra financiera, acción que produjo un caos a fin de año, momento que el venezolano recibe su pago por bono de fin de año y puede realizar sus compras decembrinas además de cancelar sus deudas. Decisión que generó un desconcierto en la dinámica ciudadana y un desequilibrio en la economía nacional. Estas apreciaciones se deben mencionar, ya que esta situación pertenece a la realidad actual de Venezuela, contexto que es relevante para plantear la problemática de esta investigación.

Con este panorama, el Ministerio del Poder Popular para la Educación (MPPPE), aplicó una Reforma del Plan de Estudios de Media General 2016-2017, como una medida para mejorar la educación en este subsistema, decisión que tomó de sorpresa a las instituciones educativas, directivos, docentes, estudiantes, padres y representantes y a la comunidad en general. Basándose en la Consulta de la Calidad Educativa realizada en el periodo 2014-2015, cuestión que no corresponde a la propuesta realizada por los actoresparticipantes, igualmente se apoyan en los fines de la educación suscrita en la Constitución de la República Bolivariana de Venezuela de 1999, la Ley Orgánica de Educación del 2009 y en el Plan de la Patria 2013-2019, enfocando éste en tres banderas importantes a su entender: a) Una pedagogía del amor, el ejemplo y la curiosidad; b) promover un clima escolar caracterizado por la convivencia y la paz y por último c) desarrollar un currículo nacional integrado y actualizado (MPPPE, 2016).

Un aspecto importante para este estudio corresponde con el incremento de las horas académicas en el área de Educación Física y su administración en tres momentos a la semana por sección, cuestión que obedece a la Ley Orgánica de Deporte, Actividad Física y Educación Física (LODAFEF, 2011), artículo 14 numeral 4: "la educación física en todo el subsistema de educación básica con una frecuencia mínima de tres sesiones por semana" (p. 8), decisión que se debe analizar, ya que de manera general el área en las instituciones educativas adolece de infraestructura -gimnasios o canchas múltiples-, cantidad de especialistas en Educación Física, actualización del programa del área, talleres de capacitación y actualización, material didáctico y la nutrición adecuada de los estudiantes son algunos elementos fundamentales para ofrecer una educación de calidad.

Para precisar los argumentos anteriores, Vargas (2016), señala la siguiente valoración:

\begin{abstract}
A una semana de que empiecen las clases de bachillerato, la confusión alrededor de la transformación curricular en educación media continúa. En agosto los voceros del gobierno comenzaron a declarar sobre este tema, pero no eran suficientemente claros sobre si en este nuevo período escolar se implementaría la adecuación del plan de estudios de manera oficial en todo el país, luego de que en 2015 se llevara la propuesta pedagógica transdisciplinaria a 68 escuelas como parte de un plan piloto. Aún no ha sido publicada alguna Resolución o Gaceta Oficial que aclare la medida. Representantes de la Asociación Nacional de Institutos Educativos Privados y la Asociación Venezolana de Educación Católica indicaron, el mes pasado, que en reuniones con el Ministerio de Educación fueron informados de que el nuevo currículo se aplicaría una vez más en un grupo limitado de planteles públicos a modo experimental para continuar evaluando el proyecto. También les afirmaron que a los colegios privados la actualización del plan de estudios llegaría en 2017-2018 (p. s/n).
\end{abstract}

Esta información corrobora el clima que existe en el subsistema de educación básica, específicamente en el nivel de educación media, ya que en el 2016 no era oficial, sino una prueba piloto, sin embargo los docentes y particularmente los de la especialidad de Educación Física están desorientado porque deben realizar ajustes en la planificación para administrar su área a futuro, aunado a los horarios del resto de los profesores.

Después de tanta incertidumbre, el Diario Correo del Orinoco (2016), presenta la siguiente información: 
El Ministerio del Poder Popular para la Educación dictó los lineamientos para el proceso de Transformación Curricular, que fueron publicados en la Gaceta Oficial de la República Bolivariana de Venezuela $N^{\circ}$ 41.044, de fecha 2 de diciembre de 2016. De acuerdo a los documentos -Resolución- DM/ $\mathrm{N}^{\circ} 0142$ y 0143, del despacho del ministro Rodulfo Pérez, se plantean referentes éticos y procesos indispensables que le dan perspectiva al quehacer educativo, como la integración, la ciudadanía participativa y protagónica, amor a la Patria, la soberanía y autodeterminación, respeto y afirmación de la condición humana, interculturalidad y valoración de la diversidad, trabajo productivo y transformación social; preservación de la vida en el planeta; la libertad y visión crítica del mundo, curiosidad e investigación (p. 2).

Con esta afirmación se aclara medianamente el panorama sobre la Reforma del proceso de Transformación Curricular planteado por el Gobierno Nacional, decisión que produjo reacciones en el escenario educativo, considerando debilidades en cuanto a los aspectos: técnicos, teóricos, conceptuales, metodológicos, infraestructura, ideológicos y prácticos.

Por su parte, Rojas \& Fermín (2016), en un artículo publicado en el Diario El Nacional, recogen las siguientes apreciaciones de personeros que se pronunciaron al respecto:

Carvajal (ob. cit.), director del doctorado en Educación de la Universidad Católica Andrés Bello (UCAB), interpreta "que si antes un profesor solo impartía una materia, ahora deberá enseñar los contenidos de las otras que agrupe el área de formación. No se opone a una reforma, incluso encuentran aspectos positivos en la propuesta, pero sí son críticos sobre los referentes ideológicos y de adoctrinamiento en los contenidos” (p. 1).

Igualmente, Ramírez, director del posdoctorado de Educación en la Universidad Central de Venezuela (UCV), opina que:

...esa podría ser una manera del gobierno para solventar el déficit de docentes, pues en lugar de contratar a tres profesores para Física, Química y Biología solo se requeriría uno para Ciencias Naturales, que arroparía esas tres asignaturas. Otro escenario sería que se mantengan educadores por materia, pero deben planificar juntos cómo enfocar un tema para englobar las disciplinas de un área de conocimiento y luego elegir quién de ellos dictará los contenidos, lo que podría generar desorganización (p. 1).

Ambos coinciden en el grave déficit de docentes especialistas. El total de profesores con título no llega a 50\% de lo que se necesita. El también miembro de la organización Asamblea de Educación recordó que en el proceso de cambio de currículo, debe ser progresivo, los profesores deben invertir tiempo mientras ocurre la transición, al respecto se pregunta ¿cómo lo van a remunerar? se hizo hincapié en que, además, la mayoría de los docentes activos no están entrenados en una práctica pedagógica transdisciplinaria, por lo que deberán pasar por un nuevo proceso formativo. El que egresó de Física en el Pedagógico a lo mejor no tiene conocimientos de Química y Biología. Tendrá que ver cómo se las arregla ante ese nuevo reto (ob. cit.).

Por su parte, Alzuru, Presidente de la Federación Venezolana de Maestros, afirmó que "no han sido consultados para esta reforma curricular”... La Asociación Venezolana de Educación Católica sostuvo una reunión con representantes del MPPPE y calificó de "positiva la iniciativa ante la deuda que existe con la actualización del currículo" (p. 1).

Asimismo, Rojas (2017), en el Diario El Nacional, complementa las aseveraciones anteriores y presenta otros académicos declarando:

Rodríguez (citada en Rojas, 2017), docente del doctorado en Educación de la Universidad Central de Venezuela e investigadora de currículo, gestión escolar y formación de docentes, señala que:

un diseño curricular debe tener un fundamento pedagógico serio que permita establecer si se trata de un modelo por competencias o por procesos, o por estándares, o si hay un desarrollo por metas o cuáles serán los perfiles de los egresados: Al no haber claridad sobre el modelo, tampoco se sabe cuáles serán los logros para saber hacia dónde va y qué va a dominar ese joven egresado tanto en contenidos como en valores...revisó los documentos del Ministerio de Educación desde 2015 sobre este proceso (se presentaron cuatro versiones) y observó que son indicaciones organizativas para las escuelas, pero no propiamente un diseño: Hay ideas rescatables, pero muchos planteamientos están desfasados. La integración por áreas es un concepto viejo en pedagogía, pero son métodos muy difíciles de poner en práctica. Hay que ver hacia dónde se está moviendo el mundo y, por ejemplo, tener un libro único es un atraso. La especialista en Educación de la UCV agrega que un diseño 
curricular no puede estar apegado a documentos como el Plan de la Patria porque es un lineamiento que se acaba a corto plazo y un currículo debe ir más allá de un período específico...(p. 2).

Por su parte, Giménez (ob. cit.), profesor de Ciencias Sociales de la Universidad Centroccidental Lisandro Alvarado e investigador del sistema escolar público en Venezuela, apunta que "la resolución es una simplificación de lo que debería ser un diseño curricular: los estudiantes de bachillerato van a estar cien años atrás de las tendencias mundiales" (p. 2).

En el caso específico del área de Educación Física, se pueden señalar las primeras apreciaciones por algunos entrevistados en el sistema: "Llegó al último año de bachillerato sin ver Química y aprobó cuarto año sin cursar nunca Educación Física", "Los profesores que anteriormente dictaban asignaturas como Dibujo Técnico, Contabilidad e Informática, ahora fueron asignados para dar clases de Ciencias Naturales, Matemáticas y Educación Física”, "Eso es como avalar la piratería”, opinó el docente. Ante el desacuerdo de algunos, William Brom jefe de la Zona Educativa para Educación Media de Distrito Capital indicó que "la primera palabra en sus títulos de grado era "Profesor", por lo que no había problema en que ellos dictaran cualquier contenido". "Educación Física antes eran 2 horas a la semana, ahora son 6 horas. Eso hizo que la plantilla de profesores no sea suficiente para todas las secciones", "Quieren darle prioridad a la Educación Física, dejando de lado aquellas materias que incentivan la lógica para formar castrados mentales” (ob. cit.).

Estas aseveraciones desmejoran la práctica docente en la especialidad de Educación Física porque ubicarán a otros especialistas a dictar esta área, además de la denigración a estos docentes por colegas de otras especialidades, padres y representantes considerando al área menos importante que otras "Grasso Error", adicionalmente existe un déficit de profesores especialistas y los que están no les aperturan concursos para su ingreso formal en las dependencias educativas. Por otro lado, el material didáctico e instalaciones deportivas están deficientes e inexistentes cuestión que limitará el proceso educativo.

Después de estas observaciones con la implementación de la reforma curricular durante el mes de diciembre 2016 y enero 2017, el Presidente de la República hace un cambio en el Gabinete Ministerial para tratar de paliar la situación. Designa al Lic. Elías Jaua como nuevo Ministro del Poder Popular para la Educación, quién en su corta experiencia en el cargo, analizó dicha situación y planteó el día 20 de enero de 2017, la suspensión del avance progresivo del plan de estudios propuesto en el artículo 8 de la Resolución 0143, la cual contempla los lineamientos del proceso de transformación curricular en todos los niveles y modalidades (Fermín, 2017).

En este sentido, Jaua (citado en Fermín, 2017), señaló "cuando hablamos de transformación académica, curricular, nuestro principal esfuerzo tiene que estar en dotar, capacitar y generar las condiciones para que ustedes educadores y educadoras, cada día tengan una mayor formación y mejores recursos pedagógicos..." (p. 1), también anunció que en los planteles privados se suspende este proceso y en los planteles oficiales que lo implementaron deben hacerle seguimiento para su revisión y modificación.

La propuesta quedó suspendida para el año escolar 2016-2017 con estas prerrogativas, sin embargo en el período 2017-2018 se volvió a intentar la reforma, sin tener éxito el gobierno, gracias al desacuerdo que mantiene la comunidad en general, dejando el plan de estudio anterior.

Con esta noticia, se abre la oportunidad de una revisión profunda en el Plan de Estudios de la Educación Media General, aunque todavía no está corregido en la nueva Gaceta Oficial. Sin embargo, este artículo toma mayor relevancia, porque en cualquier momento se decide su implementación, además que la LODAFEF estima las tres sesiones de clase de Educación Física por semana con cada grupo.

En vista de lo anterior, es relevante plantearse la siguiente interrogante ¿Cuál será la pertinencia teórica y de viabilidad de la reforma del currículo del nivel de media general en el área de Educación Física en Venezuela?

Para responder a esta inquietud, es preciso proponerse el siguiente objetivo de investigación: Analizar la pertinencia teórica y de viabilidad de la reforma del currículo del nivel de media general en el área de Educación Física en Venezuela. Asimismo plantearse los sucesivos objetivos específicos: caracterizar el diseño curricular vigente y la reforma en cuanto al área de Educación Física; describir aspectos teóricos y legales que 
fundamentan el área de Educación Física en el currículo del nivel de media general en Venezuela y analizar documentos de opinión de expertos en materia educativa sobre la reforma curricular planteada.

El producto de esta investigación documental permitirá presentar de una manera sistemática las consecuencias de la aplicación de esta reforma curricular en el sistema educativo venezolano y en especial al área de Educación Física. Para abordar esta situación, es importante conocer el estado del arte de este contexto en el ámbito internacional y nacional.

\section{Desarrollo}

En este aparte de la investigación se iniciará la revisión de estudios sistemáticos relacionados con la Educación Física y su carga horaria en el currículo escolar, partiendo de trabajos internacionales y nacionales para conocer sus experiencias en este tema.

En este sentido, Mekler (2000), realizó un estudio en Buenos Aires, Argentina sobre la Periodización del tiempo escolar en diferentes países, relacionado con una información sistematizada en relación con las decisiones adoptadas en diferentes países pertenecientes a la Comunidad Europea, a la Organización para la Cooperación y el Desarrollo Económico (OCDE) y en dos países latinoamericanos (Chile y México).

Entre los aspectos que se consideraron fueron: Duración en horas de la jornada escolar, cambios en la adecuación horaria de la jornada escolar, duración de las horas didácticas y cantidad de horas didácticas semanales, entre otras. En la mayoría de los países perteneciente a la OCDE y Latinoamérica, el inicio de la escolaridad obligatoria está fijado entre los 5 y 6 años de edad, con variaciones. Generalmente la escolaridad básica obligatoria se extiende entre 9 y 12 años de duración, con diferencias según la diversidad de los sistemas educativos. El número de jornadas de clases efectivas para un año escolar varía entre los 175 y 220 días (ob. cit.).

La mayoría de los países ofrecen una jornada para primaria que va entre 4 y 5 horas; y una jornada escolar para la secundaria inferior que se extiende entre 5 y 6 horas de clase (Cuando se define jornada diaria incluye los tiempos de recreo y traslados a diferentes aulas). Cada hora didáctica tiene una duración entre 40 a 50 minutos. Por último, resultan diversos los porcentajes en Educación Física, ya que pueden oscilar entre un $6 \%$ a un $14 \%$ representando un promedio de 45 a 55 minutos por clase, según la estructura curricular en el ciclo de secundaria inferior.

En este caso se encuentra una tendencia a la flexibilidad curricular a través de elecciones por parte de los alumnos entre ofertas de distintos deportes. Los países estudiados fueron: Alemania, Argentina, Canadá Británico, Chile, Corea, Dinamarca, España, Estados Unidos, Finlandia, Francia, Holanda, Inglaterra, Italia, Japón, México y Portugal. Documento realizado para presentarlo al Ministerio de Educación, Ciencia y Tecnología de Argentina para su estudio y análisis (Mekler, 2000).

Por su parte, Gambau (2015), investigó sobre "Las problemáticas actuales de la educación física y el deporte escolar en España, este artículo está basado en documentos de varios organismos internacionales y en el trabajo del Comité de Expertos de Educación Física de ese país” (p. 53). Gambau, inicia su investigación con la definición de la Educación Física de Calidad (EFC):

La experiencia de aprendizaje que se ofrece a los niños y jóvenes a través de las clases de educación física debe ser apropiada para ayudarles a adquirir las habilidades psicomotrices, la comprensión cognitiva y las aptitudes sociales y emocionales que necesitan para llevar una vida físicamente activa (p. 55).

Considerando estos aspectos plantea un enfoque integral con siete dominios de aprendizaje necesarios para el alumnado del siglo XXI: 1) Bienestar físico, social y emocional, 2) cultura y artes, 3) alfabetismo y comunicación, 4) perspectivas de lectura y cognición, 5) aritmética y matemáticas, 6) ciencia y 7) tecnología como competencias básicas. También precisa que existe una evidenciada conexión entre jóvenes físicamente activos y sus logros académicos; y la práctica regular de educación física de calidad y otras formas de 
actividad física puede mejorar la capacidad de atención del niño, mejorar su control cognitivo y acelerar su procesamiento cognitivo.

Es ampliamente conocida esta relación y actualmente el sedentarismo es el cuarto factor de riesgo de mortalidad más importante. La inversión pública en la educación física es ampliamente superada por los altos dividendos en ahorros para la salud y los objetivos educativos.

Gambau, continúa "el reciente documento de la UNESCO del 2015, indica que el tiempo real de aprendizaje en el currículo de Educación Física debe contener entre 120 y 180 minutos semanales, con previsión de aumentarlas, descontando el tiempo dedicado al cambio de ropa, el desplazamiento hasta instalaciones concretas, y el tiempo dedicado a otros temas como, por ejemplo, la salud" (p. 57).

Dentro del análisis de Gambau en los documentos relacionados con la EFC, señala:

El Parlamento Europeo en su informe sobre la función del Deporte en la Educación en el 2007, incluye recomendaciones a los Estados miembros a los que solicita se impongan la obligatoriedad de al menos tres horas de clase de Educación Física a la semana. No obstante, el Parlamento reconoce que lo recomendable es que los niños y niñas hagan ejercicio físico todos los días. Asimismo, la Asociación Nacional del Deporte y la Educación Física en el 2004 (National Association for Sport and Physical Education, NASPE) considera que la Educación Física debe ser diaria (p. 60).

También señala lo publicado por la Agencia Ejecutiva de la Educación Física y el Deporte en los Centros Escolares de Europa en el 2013, específicamente en España, referido al ámbito educativo, donde pone de manifiesto el escaso porcentaje de horas lectivas dedicadas en nuestro país a la Educación Física,

...las políticas educativas españolas parecen caminar en sentido contrario, a pesar de que las recomendaciones internacionales marcan la imperiosa necesidad de aumentar las horas de Educación Física en el período escolar. Por ejemplo, los países con cinco horas semanales de clase de Educación Física, como Finlandia, obtienen mejores resultados académicos en el Informe Programa para la Evaluación Internacional de Alumnos (p. 63).

La EFC desempeña un importante papel en el aprendizaje transversal. Puede contribuir de manera única al aprendizaje de otras asignaturas y a desarrollar aptitudes transferibles para apoyar el logro más allá del currículo. Desde desarrollar la comprensión de conceptos matemáticos y científicos básicos, hasta fomentar la responsabilidad medioambiental, pasando por promover el entendimiento social, histórico y cultural.

Gambau, concluye su estudio de esta manera: "La Educación Física en España está anclada y padece de una marginalidad que a simple vista no preocupa a nadie. Las políticas en materia de Educación Física van en dirección contraria a las directrices y recomendaciones internacionales y la tendencia apunta a que las prácticas deportivas de carácter competitivo e institucionalizado pretendan suplir los objetivos de una Educación Física de Calidad" (p. 65).

Este estudio evidencia la importancia de la Educación Física en el sistema educativo contemporáneo, corroborando el aumento progresivo de las horas diarias de esta asignatura, además de los beneficios que ofrece su práctica. Así como el desinterés de los gobiernos en apoyar esta asignatura.

En este mismo orden de ideas, (González, 2008), presenta en su artículo Propuesta sobre la distribución horaria de Educación Física para primer ciclo en instituciones que ofrecen más de dos estímulos semanales, específicamente en Argentina. González, señala "Es intención de la presente brindar una aproximación a este análisis en el área de Educación Física en el Primer Ciclo de la enseñanza, a partir de una experiencia donde la distribución horaria (tres clases semanales de 50 minutos) fueron administrados durante un año, los días lunes, miércoles y jueves mientras que en el año siguiente los tres estímulos fueron organizados en forma consecutiva los días martes, miércoles y jueves" (p. 1).

Se pudo determinar que la memoria motriz manifestada por los niños desde su expresión corporal hasta incluso su manifestación verbal se registró en mayor medida, en las clases consecutivas que en las no consecutivas. Eran comunes las propuestas de los mismos niños de incorporar aprendizajes del día anterior: “¿en este juego podemos pasar la pelota como hicimos ayer?” “'hoy podemos probar otra vez el juego nuevo?”. 
Las clases eran enriquecidas por el entusiasmo de los mismos alumnos al encontrar un hilo conductor en los aprendizajes en forma espontánea (ob. cit.).

En atención al estudio señalado, González, concluye que "en clases consecutivas los niños que transitan el primer ciclo capitalizan su memoria motriz en mayor medida que en las no consecutivas, relacionando 'espontáneamente' los aprendizajes anteriores con los presentes y el resultado final es que su participación en clase resulta más activa, con sentido y por lo tanto significativa. Ahora bien, el poder relacionar un aprendizaje con conocimientos anteriores (en este caso enseñados en clase) es lo que Ausubel denomina aprendizaje significativo" (p. 4).

Las razones más comunes para optar por este criterio distributivo, son las que apuntan a la necesidad de brindar un tiempo de recuperación por el esfuerzo al que supuestamente son sometidos nuestros alumnos cuando ejercitan determinadas capacidades que requerirían del funcionamiento de procesos de recuperación relacionados con el reabastecimiento del glucógenoque es el principal componente que requieren los músculos para obtener energía, entre otros (ob. cit.).

Entre las conclusiones de González se pueden señalar:

Se han detectado progresos motrices cualitativos y significativos; además de la buena predisposición con la que se presentan los alumnos a las clases consecutivas, manteniendo el estímulo de la clase anterior y ofreciendo aportes espontáneos de lo aprendido anteriormente; se ha observado que la clase resulta significativa para los niños cuando pueden relacionar motrizmente estos progresos con la clase anterior, las clases consecutivas facilitan esta relación en forma espontánea. No pretende ser una receta de cómo deben distribuirse los horarios en Educación Física, en Primer Ciclo, ni en ningún nivel de la enseñanza en particular, porque cada Institución, cada grupo, cada alumno y cada docente posee un mundo de percepciones, registros, estímulos y necesidades (p. 6).

Nuestra tarea diaria es constituirnos en el mediador que reúna estos elementos y busque los recursos, los fundamentos y las adhesiones para ofrecer cada día, cada hora y en cada minuto esa cuota de "sentido" que se refleja en la sonrisa de nuestros alumnos que ilumina nuestros corazones (p. 7).

Se considera importante el estudio anterior, ya que apunta hacia la posible práctica que se pueda implementar en Venezuela, considerando la praxis de las clases consecutivas como beneficio significativo en los educandos, por otro lado, habría que reflexionar el aspecto de la recuperación o reabastecimiento de glucógeno de un día para otro. Ahora bien, el autor no hizo referencia a otras variables como instalaciones deportivas, material didáctico, números de profesores, nutrición de los estudiantes que posiblemente en Venezuela se deben considerar.

A manera de síntesis de los antecedentes presentados anteriormente se pueden extraer: el promedio para las clases de Educación Física en los países europeos y latinoamericanos es de 40 a 50 minutos por día a la semana, además de la propuesta de la UNESCO en incrementar de 120 a 180 minutos a la semana con una tendencia en aumentar más tiempo y en el caso particular de Argentina que ha empleado y ensayado los tres momentos a la semana de dos horas de 45 minutos cada clase preferiblemente en sesiones consecutivas.

En cuanto al Parlamento Europeo en su informe sobre la función del Deporte en la Educación en el 2007 , incluye recomendaciones a los Estados miembros a que impongan la obligatoriedad de al menos tres horas de clase de Educación Física a la semana. No obstante, el Parlamento reconoce que lo recomendable es que los niños y niñas hagan ejercicio físico todos los días. Por su parte, la Asociación Nacional del Deporte y la Educación Física en el 2004 (NASPE) considera que la Educación Física debe ser diaria. Estos datos dan indicios prácticos de la dinámica de la Educación Física en varias locaciones del mundo, sin embargo cada región tiene su propia cultura y sistema educativo.

Estas investigaciones internacionales dan luz para analizar la situación curricular en Venezuela, no obstante se deben revisar los estudios que se han generado en el país para corroborarla con otras naciones.

En relación a lo anterior, (Camacho, 2014), realizó un artículo titulado La Educación Física en el nuevo diseño curricular, presentando un análisis sobre la implementación del Diseño Curricular Bolivariano 2007 y su impacto en el área de Educación Física en el estado Carabobo, además pretende contribuir al debate 
planteado actualmente sobre el sentido de la educación y las transformaciones que amerita, visto desde el papel fundamental que protagoniza la Educación Física, el Deporte y la Recreación dentro del currículo aún en discusión.

Entre el análisis realizado en la Zona Educativa del estado Carabobo, se pueden mencionar: En el subsistema de Educación Primaria, aun no se implementan las tres (3) sesiones de clase semanal (exigido en la Ley Orgánica de Deporte, la Actividad Física y la Educación Física, Art. 14 numeral 3 y 4); para lo cual se necesita una cantidad considerable de recurso humano (docentes); con los que se cuenta en el Estado, según el último Censo de Profesores de Educación Física de la División de Deportes de Zona Educativa del 2012, donde para ese año se contaba con 400 profesores egresados de las diferentes universidades locales y de Estados vecinos, que esperan por ingreso al campo laboral, a esto se suma los que han egresado hasta la fecha. Para el año 2013 se presenta de parte de la Zona Educativa Carabobo ante el MPPE, la propuesta de implementación de las tres sesiones de clase de Educación Física en primaria (ob. cit.), donde Camacho recomienda lo siguiente:

Actualmente en todos los niveles y modalidades existe un total de 241 planteles sin docentes especialistas en el área de Educación Física, Deporte y Recreación; además tomando en cuenta que muchos de esos planteles necesitan más de un docente por el número de matrícula, lo que duplicaría y hasta triplicaría estas necesidades, un ejemplo claro en Educación Media General es que existen 208 planteles donde 60 no tienen especialistas de Educación Física; se ve como prioridad, ingresar a los versados necesarios en las vacantes disponibles (p. 2).

Una vez resueltas las necesidades de docentes en los planteles, se puede pensar en implementar el aumento de las sesiones de clase, con una frecuencia de tres (3) momentos a la semana, aunque la Ley explica que esto debe cumplirse hasta el subsistema de Educación Básica, lo que implica el nivel de la Educación Media General y Media Técnica.

Para el Currículo Básico Bolivariano, en el subsistema de Media Técnica la propuesta para el currículo en discusión, es implementar las tres (3) sesiones de clase semanal, considerando que cuarenta y cinco minutos $\left(45^{\prime}\right)$ una vez a la semana, no es suficiente para continuar con el desarrollo de las capacidades condicionales, cognitivas y coordinativas; además de fortalecer valores y hábitos hacia la práctica de la actividad física y el deporte para preservar la salud, adquiridos durante las etapas anteriores. Camacho concluye:

el futuro de la educación y del país dependen en gran medida de la conformación de un currículo, producto del trabajo de un equipo multidisciplinario, de las experiencias del educador que ama su profesión; del estudiante que espera se le dé la oportunidad de ser productivo dentro de una sociedad cada vez más cambiante y exigente; de la familia como núcleo importante de la misma, y de todos los actores y dolientes que apostamos a un currículo centrado en el educando y la sociedad para cambiar al mundo, por algo Nelson Mandela aseveró "la educación es el arma más poderosa que se puede usar para cambiar el mundo" (p. 4).

En síntesis, este estudio se realizó en el 2014 sobre la Educación Física en el Diseño Curricular Bolivariano 2007, analizando su impacto en los especialistas del área, ya en ese momento se detectaban las debilidades que existen con el déficit de profesionales de la Educación Física, además de la falta de apertura de concurso de ingreso al sistema educativo nacional, todavía existe la figura de docentes interinos cuando se tienen cargos vacantes en las instituciones educativas en el estado Carabobo como en otras regiones del país.

Aunado a esto, Méndez (2015), tituló en el Diario El Nacional Reforma curricular de Educación Media exige una alta inversión económica, comenta la autora, siguiendo "la aplicación del turno integral obliga a incrementar el número de docentes, construir nuevos planteles y garantizar la dotación de insumos y material didáctico" (p. s/n).

Entre sus entrevistados, Mundó (citada en Méndez, 2015), "aplaudo la iniciativa oficial de reformar la Educación Media. El problema es que estamos en una crisis económica y esa propuesta exige una alta y sostenida inyección de fondos para construir nuevos planteles, pagar mejores salarios a un mayor número de docentes. Se requiere una planificación financiera" (p. s/n).

Mundó (ob. cit.) agregó: 
...en la gestión del exministro de Educación, Aristóbulo Isturiz, se anunció la activación de los Liceos Bolivarianos con turno integral. Sin embargo, pocos son los liceos que funcionan con esa metodología, y no hay registros oficiales sobre sus avances pedagógicos. La nueva propuesta del MPPPE también implica ofrecer a los alumnos desayuno y almuerzo, un servicio costoso que presenta fallas en las Escuelas Bolivarianas. Ese punto se debe revisar muy bien. En los liceos la pirámide socioeconómica indica que quienes están en los años superiores son menos pobres con respecto a los alumnos que se inician. Entonces se estaría subsidiando a un sector que no lo necesita afectando a los niños de las escuelas (p. s/n).

Asimismo, Herrera (citado en Méndez, 2015), director del Centro de Investigaciones Culturales y Educativas (CICE), señaló: "El nuevo esquema implica que el máximo de horas de un profesor debe concentrarse en un solo liceo. Estaríamos estimando 16 horas de clases, y 20 horas administrativas, como sucede en algunos países de Europa. De entrada eso requiere incrementar en 30\% la nómina docente. Este cambio demanda una precisa planificación académica" (p. s/n).

De acuerdo a las apreciaciones de los académicos e investigadores del área educativa, se puede interpretar que la puesta en marcha de esta reforma requiere de un esfuerzo financiero y administrativo por parte del gobierno nacional, cuestión que para ese 2015 era complicado ahora para este 2018 es insostenible.

Por su parte, (Lozada, 2016), publicó en Efecto Cocuyo, el siguiente artículo "El nuevo currículo escolar no tiene asignaturas, sino 'áreas' de formación. Al respecto, inició con una aseveración del MPPPE en el 2015-2016, la transformación al currículo educativo de Educación Media se implementó en este año escolar en 68 planteles modelos" (p. s/n).

Al respecto, Aguilar (citada en Lozada, 2016), Directora Nacional de Fe y Alegría, señaló que "es de cambio profundo pero que falta afinar unos detalles de implementación, como infraestructuras adecuadas, contratación de los docentes y dotación de alimentación, pues implicaría un cambio de horario" (p. s/n).

La propuesta del nuevo currículo no incluye asignaturas: son ejes de aprendizaje, que permitan garantizar la continuidad durante los cinco años, especificando que deben estar transversalizados con los cinco objetivos históricos del Plan de la Patria en un tejido interdependiente. Estos ejes son: Lenguas, Culturas y Comunicación (LCC), Memoria, Territorio y Ciudadanía (MTC), Matemática, Ciencias Naturales, Lenguas Extranjeras (LE), Educación para el Trabajo (EPT) y Educación Física. De primero a tercer año, cada uno tendría 6 horas semanales, menos Memoria, Territorio y Ciudadanía, que tendría 8. En cuarto y quinto año, Lenguas, Culturas y Comunicación tendrían 4 horas, Memoria, Territorio y Ciudadanía 6, al igual que Lenguas Extranjeras, Matemática y Educación Física, mientras que Educación para el Trabajo tendría 4 horas y Ciencias Naturales 12 (ob. cit.).

En esta transformación curricular se agregó los llamados Grupos Estables o grupo de creación, recreación y participación, es decir, grupos culturales, clubes deportivos, estudiantinas, cineclubes o corales, organizados bien sea por plantel o por circuito escolar que integran el currículo. De esta manera, no serán vistos como actividades complementarias ni extra-curriculares, porque todo lo que las escuelas y liceos hacen para la formación integral de nuestros niños, niñas, adolescentes, jóvenes, adultos y adultas, es currículo (ob. cit.).

Asimismo, asegura que tiene lógica el pensar en un nuevo currículo para actualizar y permitir innovación en las aulas de clase. Tener un currículo de 1969 es un hándicap para la educación. Sin embargo, dice que el Gobierno argumenta que este currículo es el resultado de la Consulta Nacional por la Calidad Educativa de 2014, pero que estos no fueron los resultados de la misma. Lozada (ob. cit.), agrega que en la consulta "Un $53 \%$ dijo que la formación docente es el elemento más importante, y solo el $8 \%$ dijo que era necesario un cambio curricular" (p.s/n).

En este artículo se pueden observar las diferentes opiniones de los actores de esta reunión, sin embargo las deficiencias en infraestructura, personal docente, alimentación, planificación son comunes a las apreciaciones de otros investigadores, ahora bien, si el Ministerio lo aplicó en Instituciones Educativas, ¿̇cuáles fueron los resultados? Continuando con el tema central de esta investigación, relacionado con la Pertinencia Teórica y de Viabilidad de la Reforma del Currículo del Nivel de Media General en el Área de Educación Física, se puede observar en la Figura 1, un ejemplo de horario de clases de 4to. o 5to. año propuesto por el MPPPE. 
Figura 1. Ejemplo de horario de Media General. Tomado: Lozada (2016) Diario Efecto Cocuyo.

\begin{tabular}{|c|c|c|c|c|c|}
\hline $7: 00-7: 45$ & \multicolumn{5}{|c|}{ Hora Civica y Desavuno } \\
\hline $7: 45 \cdot 9: 15$ & LCC & LCC & $\begin{array}{l}\text { Lab Fis/Lab } \\
\text { Bie }\end{array}$ & LE & EPT/EPT \\
\hline $9: 20 \cdot 10: 50$ & $\mathrm{M}, \mathrm{T}$ y $\mathrm{C}$ & $\mathrm{M}, \mathrm{T} \times \mathrm{C}$ & $M, T \times C$ & Biologia & EPT/EPT \\
\hline $11: 00-12: 30$ & Matemática & Fisica & Matemaitica & Matemática & Quimica \\
\hline $12: 30-1: 30$ & \multicolumn{5}{|c|}{ Almuerzo } \\
\hline $1: 30-3: 00$ & $\begin{array}{l}\text { Lab Qui/lab } \\
\text { Bio }\end{array}$ & Efecto & Cocuyo & Guiatura & Ed. Fisica \\
\hline $3: 05 \cdot 4: 35$ & Ed. Fisica & \multirow[t]{2}{*}{$\begin{array}{l}\text { Grupos } \\
\text { estables }\end{array}$} & Ed. Fisica & $\begin{array}{l}\text { Lab Fis/Lab } \\
\text { Qui }\end{array}$ & \multirow[t]{2}{*}{$\begin{array}{l}\text { Grupos } \\
\text { estables }\end{array}$} \\
\hline $4: 40-5: 25$ & & & & & \\
\hline
\end{tabular}

Claramente se puede observar el horario y la frecuencia de las clases de Educación Física, aspecto relevante para este estudio. La aplicación de esta reforma en el área representa un valor agregado para los especialistas y un reto, ya que tradicionalmente son dos (2) o una (1) hora de $45^{\prime}$ por cada sección.

También hay que considerar el escenario legal, el MPPPE (2013), se refiere a la Cláusula $N^{\circ} 17$ de la VII Contratación Colectiva 2013-2015, que describe, "las clases prácticas como talleres, laboratorios, formación para el trabajo, educación para las artes y Educación Física la relación de docente-alumnos por aula debe ser 15 estudiantes" (p. 15), lo que representa que cuando existan secciones con más de quince (15) educando se divide en dos subgrupos de la misma sección, cada subgrupo le corresponde un profesor, esto generará una nueva estructura en la distribución de los horarios de los profesores así como la planificación de las clases, la organización del espacio físico (cancha y/o gimnasio) y el material deportivo.

En este particular, es preciso analizar de una manera sistemática los elementos que se presentaron en los párrafos anteriores. En el caso de la carga horaria de los profesores especialistas en Educación Física, un docente que tenga asignada 36 horas semanales académicas debe administrar en los actuales momentos (2018) aproximadamente 17 secciones de 15 alumnos o alumnas por $2 \mathrm{~h}$ de $45^{\prime}$, lo que representaría $34 \mathrm{~h}$ docentes más $2 \mathrm{~h}$ de guiatura u otra actividad. En este sentido, al aplicarle la reforma del Plan de Estudio 2017-2018, donde se proponen tres momentos con cada sección tendría que administrar aproximadamente 6 secciones quedando 11 secciones sin docentes o se tendrían que incorporar 2 docentes especialistas para cubrir estas horas, o sea por cada profesor de $36 \mathrm{~h}$ se tendrían que contratar 2 docentes para el resto de las secciones.

Adicionalmente el espacio físico (cancha o gimnasio) debe considerarse porque van a estar más docentes y secciones en los ambientes de aprendizajes, en el caso cuando la Institución tiene varias instalaciones tal vez no habría problemas, sin embargo la gran cantidad de liceos no poseen estas condiciones, lo que generaría una situación problemática.

Aunado a lo anterior, se suma el material deportivo, considerando que actualmente las Instituciones adolecen de este recurso didáctico, ya que el Ministerio es el que distribuye y equipa de este material a los liceos y escuelas nacionales, lo cual no lo hace periódicamente. Es importante acotar que la inversión financiera de este recurso es costosa, lo que fundaría un presupuesto generoso, considerando además que este material no tiene mucho tiempo de vida útil por más que se cuide, pero el uso constante lo deteriora, agregando que la cantidad de momentos va a aumentar. En este caso, se debe repensar el cómo se podría subsanar este aspecto.

En otro particular, se puede revisar la Colección Bicentenario del 2011 que se les ha entregado a los estudiantes de Educación Secundaria (1ro. a 5to. año) por parte del gobierno como material de referencia para las clases en el Sistema de Educación Bolivariana, en este sentido se puede destacar que las áreas y textos reflejados son: Lengua y Literatura, Matemáticas, Ciencias Naturales, Educación Artística, Ciencias Sociales y Ciencia de la Tierra, observando en esta publicación y distribución que han obviado el Área de Educación Física, razones que se desconocen y que forma parte de este análisis sobre la pertinencia y viabilidad de la reforma del currículo de la media general en esta especialidad. 
Adicionalmente, se puede mencionar que en el pensum de estudios de Secundaria o tercera etapa de Educación Básica o Media General contempla el área de Educación Física en el primer año, luego para el segundo año a quinto año de Media General se denomina Educación Física y Recreación, ahora en la Educación Media Técnica no aparece, cuestión que está en contra de la LOE (2009) y LODAFEF (2011), vulnerando su obligatoriedad en todos los niveles y modalidades (Estrada, 2013). Agregando otro elemento para este análisis, se puede señalar a Estrada (ob. cit.), quién describe:

\begin{abstract}
Para el año escolar 2000-2001 algunas escuelas que contaban con Preescolar, Primera y Segunda Etapa de Educación Básica (ahora nivel de Educación Inicial y Primaria) fueron incluidas en un plan piloto pasando a llamarse Escuelas Bolivarianas, Centro de Educación Inicial o Simoncitos así mismo, en el año escolar 2002- 2003 un grupo de Unidades Educativas que contaban con la III Etapa de Educación Básica y Media Diversificada y Profesional se transformaron en Liceos Bolivarianos o Escuelas Técnicas Robinsoniana. Una reseña importante a destacar, es aquella referida a la dualidad de modelos organizativos en el sistema educativo, es decir, la Educación Básica, presenta dos modelos organizativos, por un lado se encuentran los Liceos Bolivarianos, Escuelas Técnicas Robinsoniana, las Escuelas Bolivarianas y Centro de Educación Inicial, por el otro los Ciclo Básico Común, la Educación Media Diversificada, la Educación Media Profesional, las Escuelas Técnicas Industriales y Comerciales, Unidades Educativas, Grupos Escolares y Escuelas Básicas (estas últimas pertenecientes al modelo curricular y pedagógico organizado por la Ley Orgánica de Educación de 1980 y que se mantiene vigente debido a que no ha existido una reforma curricular de los programas que se aplican en la Educación Secundaria) (p. 32).
\end{abstract}

Estas ambigüedades son las que generan incertidumbres en el escenario educativo venezolano, es por esto que se debe considerar este estudio como insumo concreto para vislumbrar el panorama real de la Educación venezolana, específicamente en el área de Educación Física.

\title{
Metodología
}

Para este artículo se recurrió a una investigación documental basada en la recopilación de estudios previos en el ámbito internacional y nacional para conocer el estado del arte de la situación planteada, además de documentos oficiales, legales y teóricos referidos a la pertinencia teórica y de viabilidad de la reforma del currículo en el área de Educación Física, asimismo se presentaron y analizaron opiniones de investigadores reconocidos y dedicados al estudio del sistema educativo venezolano a través de los medios de comunicación físico y digital.

Para su análisis se utilizó la técnica de contrastación de autores, documentos y experiencias, incluyendo el aporte intersubjetivo de los responsables de este artículo como conocedores y especialistas en el área de educación y de Educación Física.

\section{Resultados y Discusión}

Después de hacer una revisión exhaustiva a través de la investigación documental sobre la pertinencia teórica y de viabilidad de la reforma del currículo del nivel de media general en el área de Educación Física en Venezuela, se puede inferir:

Es importante señalar que el Plan de Estudio establece las asignaturas, áreas y similares de cada campo de estudio, especialidad y mención, de acuerdo con lo señalado en la normativa legal y a las exigencias de formación general y específica determinadas en los respectivos perfiles de sus egresados. Asimismo, determina la correspondiente distribución de carga horaria semanal, con los énfasis requeridos por las diferentes especialidades y menciones.

En este sentido, la propuesta del Ministerio del Poder Popular para la Educación para la Reforma del Plan de Estudio en el 2016-2017 y 2017-2018 definitivamente no logró su instauración por la presión y demandas de las organizaciones educativas, asociación civil, gremios, académicos, investigadores en el área de currículo, periodistas, docentes y padres y representantes. Sin embargo, el ente rector implementó la propuesta en varias 
instituciones educativas a manera de ensayo para determinar los resultados de su aplicación y presentar el efecto que generó al subsistema objeto de estudio.

Ahora bien, en lo que respecta al Área de Educación Física, los estudios empíricos y opiniones de expertos referidos en este artículo señalan la importancia de esta disciplina académica en cuanto al beneficio que promueve para la salud integral del individuo y sus efectos en el desarrollo biológico, psicológico, social, cognoscitivo, afectivo y formación en valores que el ciudadano requiere. Coadyuvando además, a la práctica deportiva sistemática en el ámbito escolar para formar la generación de relevo de los atletas de alto rendimiento.

Las experiencias en los países europeos y en algunas naciones suramericanas, aún se mantiene la sesión de clases de 40 a 50 minutos por semana, sin embargo la UNESCO propone aumentar la cantidad de tiempo de 120 a 180 min por semana como una política sociocultural de los pueblos, otros exigen que la Educación Física se administre todos los días. Los países con cinco horas semanales de clase de Educación Física, como Finlandia, obtienen mejores resultados académicos según el Informe del Programa para la Evaluación Internacional de Alumnos (PISA).

También existe un estudio donde las clases se administren tres veces por semana de 90 min cada una, donde se recomienda que sea de manera consecutiva, lo cual generaría un aprendizaje significativo en la memoria motriz y verbal en el educando, esta experiencia sería un elemento importante para su aplicación en Venezuela. Asimismo refiere una alimentación balanceada para la recuperación relacionada con el reabastecimiento del glucógeno, principal componente que requieren los músculos para obtener energía.

Otro elemento relevante dentro del estudio, se refiere a las cuatro teorías que prefieren los estudiantes (chicas y chicos) de secundaria para una Educación Física de Calidad, entre ellas: Salud (la actividad física para la vida), rendimiento (desarrollo del deporte), recreativa (actividades lúdicas) y expresión (la estética del cuerpo).

Por otra parte, en las investigaciones consultadas en el ámbito internacional no se encontraron evidencias importantes referidas a: actualización de los programas del área; calidad, cantidad y remuneración del docente; instalaciones y material deportivo; alimentación, estos elementos son señalados en las investigaciones nacionales así como también en los artículos de prensa donde puntualizan estas debilidades del currículo venezolano.

\section{Conclusiones}

En este sentido se puede concluir que el área de Educación Física en el Sistema Educativo venezolano requiere de mucha atención, si bien es cierto que el aumento de horas y sesiones de clase para los estudiantes es un paso importante para los especialistas, porque pueden desarrollar con mayor fluidez las competencias, las destrezas y habilidades de los educandos, pero también hay que detenerse en ver su viabilidad, ya que existen problemas estructurales que limitan esta acción, entre ellas: incertidumbre sobre la implementación del plan de estudios, modernizar y transformar los programas didácticos (oficial) del área, diseñar cursos, talleres de actualización y capacitación a los especialistas sobre las estrategias de enseñanza-aprendizaje, promoción de acceso a la carrera de formación docente por el déficit en el área, apertura de concursos de oposición para los cargos vacantes, actualizar el plan de estudios y el currículo de los estudios de pregrado, remuneración y beneficios socioeconómicos acorde, instalaciones deportivas idóneas, material deportivo suficiente y de calidad, garantizar una alimentación balanceada y nutritiva para que los estudiantes puedan realizar las actividades físicas, deportivas y recreativas adecuadamente.

Adicionalmente se puede señalar que es pertinente realizar reformas en cualquier área de interés en un país, pero debe haber conciencia y racionalidad universal para modernizar y transformar el currículo de cualquier sistema educativo, apartando la ideologización política como eje transversal en la formación de sus ciudadanos. 
Después de este resultado sistemático se hace un exhorto al gobierno nacional y al ente rector de la educación en Venezuela a una reflexión profunda para atender de manera urgente los requerimientos que se han presentado en esta investigación y así conducir al país en el rumbo de una educación de calidad.

\section{REFERENCIAS}

Camacho, M. (2014). La Educación Física en el nuevo diseño curricular. Ciencias de la Educación de la Universidad de Carabobo, 24(44).

Correo del Orinoco. (8 de Diciembre de 2016). En Gaceta Oficial: Publicados lineamientos para el proceso de transformación curricular. Diario Correo del Orinoco, p. 2.

Estrada, Y. (2013). Estudio exploratorio documental de la aplicación del artículo 112 del reglamento de la ley orgánica de educación dentro de los procesos de planificación y evaluación en la educación media general y educación media técnica. Implicaciones tecnoprácticas. Trabajo de Ascenso no publicado. Universidad Nacional Abierta.

Fermín, M. (21 de Enero de 2017). Ministerio de Educación suspendió nuevo plan de estudios para media. El Nacional, p. 1.

Gambau, V. (2015). Las problemáticas actuales de la educación física y el deporte escolar en España. Revista Española de Educación Física y Deportes, 411, 53-69.

González, P. (2008). Propuesta sobre la distribución horaria de Educación Física para primer ciclo en instituciones que ofrecen más de dos estímulos semanales. Revista Digital efdeportes, 13(121). Recuperado de http://www.e fdeportes.com/

Lamogglia de Ramírez, F. (2012). Capacitación del profesorado venezolano. Experiencia de consolidación de los círculos de acción docente en la educación básica. (Tesis doctoral). No publicada. Universidad de Valladolid, España.

Ley Orgánica de Deporte, Actividad Física y Educación Física (2011). Gaceta Oficial N 39.741 del 23 de agosto de 2011.

Linarez, R. (2016). 2016: Venezuela, contexto político actual. Portal de Overblog Publicado en 28 marzo 2016. [Consultado 2016, Diciembre 28].

Lozada, M. (21 de Agosto de 2016). El nuevo currículo escolar no tiene asignaturas, sino "áreas de formación”. Efecto Cocuyo.

Meckler, V. (2000). Periodización del tiempo escolar en diferentes paises. Ministerio de Educación, Ciencia y Tecnología, Subsecretaría de Educación y Programa de Gestión Curricular y Capacitación. Buenos Aires.

Méndez, G. (Abril, 2015). Reforma curricular de Educación Media exige una alta inversión económica. Recuperado de http://www.elnacional.com

Ministerio del Poder Popular para la Educación. (2013). VII Contratación Colectiva 2013-2015. Caracas: Autor.

Ministerio del Poder Popular para la Educación. (2016). Orientaciones Pedagógicas 2016-2017. Caracas: Autor.

Organización de las Naciones Unidas (1948). Declaración internacional de los derechos humanos. Madrid.

Organización de las Naciones Unidas (Septiembre, 2015). La ONU califica la actual crisis mundial de la más grave de su historia. Recuperado de https://mundo.sputniknews.com/mundo.

Rojas, G. \& Fermín, M. (9 de Diciembre de 2016). Propuesta de reforma curricular requerirá nueva formación docente. El Nacional.

Rojas, G. (21 de Enero de 2017). Proceso de transformación curricular. El Nacional.

Vargas, C. (Diciembre, 2016). Ministerio de Educación: Año escolar comienza con nuevo currículo en escuelas públicas. Recuperado de http://www.diariocontraste.com. 